\title{
IMPORTÂNCIA DAS INTERACCÕES CULTIVARES X LOCAIS E CULTIVARES X ANOS NA AVALIAÇÃO DE MILHO NA SAFRINHA ${ }^{1}$
}

\author{
FLÁVIA MARIA AVELAR GONÇALVES², SAMUEL PEREIRA DE CARVALHO ${ }^{3}$, MAGNO ANTONIO PATTO RAMALHO ${ }^{4}$ \\ e LUIZ ANDRÉ CORRÊA ${ }^{5}$
}

\begin{abstract}
RESUMO - Com o objetivo de verificar a importância da interação cultivares $\mathrm{x}$ locais e cultivares $\mathrm{x}$ anos e de identificar entre os híbridos já disponíveis os que sejam mais adaptados e estáveis para o cultivo em safrinha, foram estimados os parâmetros de adaptabilidade e estabilidade a partir de dados obtidos em experimentos de avaliação de cultivares de milho na safrinha, nos anos de 1993, 1994 e 1995. Esses experimentos foram conduzidos nos Estados de São Paulo, Goiás e Paraná. O delineamento utilizado foi em blocos casualizados com três repetições. Constatou-se que a magnitude da variância da interação cultivares com anos dentro de locais foi mais expressiva do que entre locais, o que indica a necessidade de que as avaliações de cultivares na safrinha sejam realizadas num maior número de anos. As cultivares diferiram quanto à adaptabilidade e estabilidade de produção de grãos. Os materiais que se destacaram como mais adaptados e estáveis foram o híbrido simples Zeneca 8452, o híbrido triplo C 805 e o híbrido duplo Agromen 2012. Já os híbridos duplos BR 201, Planagri 411, AG 303 e C 125 foram os que mostraram menor adaptação e maior instabilidade.
\end{abstract}

Termos para indexação: interação genótipos x ambientes, estabilidade, adaptabilidade, melhoramento.

\section{IMPORTANCE OF CULTIVARS X LOCATIONS AND CULTIVARS X YEARS INTERACTIONS IN MAIZE EVALUATION IN THE "SAFRINHA"}

\begin{abstract}
Aiming to check the importance of cultivars $x$ locations and cultivars $x$ years interactions, and to identify among the available hybrids those which are most adapted and stable for cultivation in "safrinha", the parameters of adaptability and stability were studied using the data previously obtained in some evaluation trials of maize cultivars in the "safrinha" during the years of 1993, 1994 and 1995. The experiments were conducted in the states of São Paulo, Goiás and Paraná, in Brazil. The experimental design utilized was in randomized blocks with 3 replications. It was verified that the magnitude of variation of the interactions of the cultivars $x$ years inside the localities was more prominent than that by localities, indicating the necessity that evaluations of the cultivars in the harvesting period be accomplished in more years. The cultivars differed based on adaptability and stability of grain yield. The materials that distinguished themselves as more adaptable and stable were the simple hybrid Zeneca 8452, the triple hybrid C 805 and the double hybrid Agromen 2012. Now, the double hybrids BR 201, Planagri 411, AG 303 and C 125 were those that showed lesser adaptability and higher instability.
\end{abstract}

Index terms: genotype x environment interactions, stability, adaptability, breeding.

${ }^{1}$ Aceito para publicação em 6 de novembro de 1998. Extraído da Dissertação de Mestrado do primeiro autor.

${ }^{2}$ Eng a Agrạ, aluna do Curso de Pós-Graduação, UFLA, Caixa Postal 37, CEP 37200-000 Lavras, MG. E-mail: avelar@ufla.br

${ }^{3}$ Eng. Agr., Dr., Prof. Adjunto, Dep. de Agricultura, UFLA. E-mail: samuelpc@ufla.br

${ }^{4}$ Eng. Agr., Dr., Prof. Titular, Dep. de Biologia, UfLA. E-mail: magnoapr@ufla.br

${ }^{5}$ Eng. Agr., M.Sc., Embrapa-Centro Nacional de Pesquisa de Milho e Sorgo (CNPMS), Rodovia MG 424, Km 65, Caixa Postal 151, CEP 35701-970 Sete Lagoas, MG. E-mail: lacorrea@cnpms.embrapa.br

\section{INTRODUÇÃO}

Na região Centro-sul do Brasil, onde se concentra a maior parte da produção de milho, há duas safras distintas: a da época normal, cuja semeadura se estende do final de setembro até meados de dezembro, e a safrinha, com semeadura nos meses de janeiro a abril, dependendo da região.

A safrinha começou por iniciativa dos agricultores, especialmente no Estado do Paraná, na década de 1980, visando a uma opção de sucessão à cultura 
da soja semeada no verão. Com o decorrer dos anos, a importância dessa prática foi crescente e se estendeu para outros estados.

As pesquisas em melhoramento genético, tanto na empresa privada quanto na pública, concentraram-se na época normal. Com o incremento da importância da safrinha, a primeira contribuição dada pelas empresas de sementes foi avaliar as cultivares disponíveis, desenvolvidas para a época normal de semeadura sob a nova condição ambiental.

Ante a diversidade de ambiente em que o cultivo de milho nessa época é submetido, é esperada a ocorrência de uma forte interação cultivares $\mathrm{x}$ ambientes. Para atenuar os efeitos dessa interação, a principal opção é identificar cultivares que sejam mais estáveis (Cruz \& Regazzi, 1994). Para identificação de cultivares mais estáveis dispõe-se atualmente de várias metodologias (Eberhart \& Russel, 1966; Lin \& Binns, 1988; Cruz et al., 1989; Annicchiarico, 1992), e a opção por uma delas está na dependência da informação fornecida e, sobretudo, da facilidade de análise e interpretação dos resultados.

Foram utilizados os dados dos experimentos de avaliação de cultivares de milho em safrinha, coordenados pela Embrapa-Centro Nacional de Pesquisa de Milho e Sorgo, conduzidos em diversos locais durante os anos de 1993, 1994 e 1995. Este trabalho foi realizado com os objetivos de verificar a importância das interações cultivares $\mathrm{x}$ locais e cultivares $\mathrm{x}$ anos, e identificar, entre os materiais disponíveis, os que fossem mais adaptados e estáveis para o cultivo em safrinha.

\section{MATERIAL E MÉTODOS}

Foram utilizados, neste trabalho, dados dos ensaios de avaliação de cultivares de milho-safrinha dos anos de 1993, 1994 e 1995, da Embrapa-Centro Nacional de Pesquisa de Milho e Sorgo. Os experimentos foram conduzidos nos Estados de Goiás, Paraná e São Paulo, nas localidades especificadas na Tabela 1 . As épocas de semeadura variaram de janeiro a maio.

Foram efetuadas análises conjuntas da produtividade de grãos, ajustados para o estande ideal (50 plantas por parcela) e umidade de 15\%, para os biênios 1993/94,
1993/95 e 1994/95, e para o triênio 1993/94/95, utilizando as cultivares e locais comuns nesses anos. No biênio de 1993/94, utilizaram-se 25 cultivares; no biênio 1993/95, 15; no biênio 1994/95, 26; no triênio 1993/94/95, 14. Em todos biênios foram analisados os dados de seis locais, e no triênio, cinco. Utilizou-se o seguinte modelo, sendo considerado como fixo o efeito da média e das cultivares:

$\mathrm{y}_{\mathrm{ijkq}}=\mathrm{m}+\mathrm{g}_{\mathrm{i}}+\mathrm{l}_{\mathrm{k}}+\mathrm{a}_{\mathrm{q}(\mathrm{k})}+\mathrm{b}_{\mathrm{j}(\mathrm{kq})}+(\mathrm{gl})_{\mathrm{ik}}+(\mathrm{ga})_{\mathrm{iq}(\mathrm{k})}+\mathrm{e}_{\mathrm{ijkq}}$,

em que:

$\mathrm{y}_{\mathrm{ijkq}}$ : valor observado da cultivar "i $\mathrm{i}$ " na repetição "j", no local "k", no ano "q";

m: média geral;

$\mathrm{g}_{\mathrm{i}}$ : efeito da cultivar "i", com "i" $=1,2, \ldots, \mathrm{n}$;

$\mathrm{a}_{\mathrm{q}(\mathrm{k})}$ : efeito de ano "q" no local "k", com "q" $=1,2, \ldots, \mathrm{m}$; $1_{k}$ : efeito de local " $k$ ";

$b_{j(k q)}$ : efeito de bloco "j", dentro do local "k" e do ano "q"; $(\mathrm{ga})_{\mathrm{iq}(\mathrm{k})}$ : efeito da interação da cultivar "i" com o ano "q" dentro do local "k";

(gl) $)_{\mathrm{ik}}$ : efeito da interação da cultivar "i" com o local "k";

$\mathrm{e}_{\mathrm{ijkq}}$ : erro experimental, correspondente à interação da cultivar "i" com o bloco "j", dentro do local "k" no ano "q".

A partir desse modelo, foram estimados, para o triênio, os componentes da variação das interações cultivares $x$ anos/locais e cultivares $x$ locais .

Para estimar os parâmetros de estabilidade foi utilizada metodologia de Lin \& Binns (1988), que se baseia no princípio de que nas avaliações de cultivares o que se procura é o material com desempenho próximo do máximo para a maioria dos ambientes. Para avaliar esse tipo de estabilidade, utilizou-se a seguinte expressão:

TABELA 1. Coordenadas geográficas e altitude dos locais dos experimentos de avaliação de cultivares de milho-safrinha.

\begin{tabular}{lccc}
\hline Locais & \multicolumn{2}{c}{$\begin{array}{c}\text { Coordenadas } \\
\text { geográficas }\end{array}$} & $\begin{array}{c}\text { Altitude } \\
(\mathrm{m})\end{array}$ \\
\cline { 2 - 3 } & Latitude & Longitude & \\
\hline Santa Cruz das Palmeiras, SP & $21^{\circ} 50^{\prime} \mathrm{S}$ & $47^{\circ} 16^{\prime} \mathrm{W}$ & 644 \\
Cravinhos, SP & $21^{\circ} 20^{\prime} \mathrm{S}$ & $47^{\circ} 44^{\prime} \mathrm{W}$ & 782 \\
Goiatuba, GO & $18^{\circ} 00^{\prime} \mathrm{S}$ & $49^{\circ} 21^{\prime} \mathrm{W}$ & 630 \\
Paulínia, SP & $22^{\circ} 45^{\prime} \mathrm{S}$ & $47^{\circ} 10^{\prime} \mathrm{W}$ & 564 \\
Barretos, SP & $20^{\circ} 34^{\prime} \mathrm{S}$ & $48^{\circ} 34^{\prime} \mathrm{W}$ & 552 \\
Guaíra, SP & $20^{\circ} 18^{\prime} \mathrm{S}$ & $48^{\circ} 18^{\prime} \mathrm{W}$ & 490 \\
Jacarézinho, PR & $23^{\circ} 09^{\prime} \mathrm{S}$ & $49^{\circ} 58^{\prime} \mathrm{W}$ & 435 \\
\hline
\end{tabular}

Fonte: Enciclopédia dos Municípios Brasileiros (1958). 
$P_{i}=\sum_{j=1}^{n}\left(Y_{i j}-M_{j}\right)^{2} / 2 n$

em que:

$\mathrm{P}_{i}$ : índice de estabilidade da cultivar "i", ou seja, mede o desvio da produtividade de uma dada cultivar em relação ao máximo, em cada um dos “j” ambientes;

$\mathrm{Y}_{\mathrm{ij}}$ : produtividade da cultivar "i" no ambiente "j";

$\mathrm{M}_{\mathrm{j}}$ : produtividade da cultivar com resposta máxima entre todos as cultivares no ambiente " $\mathrm{j}$ ";

n: número de ambientes.

Essa expressão foi desdobrada em:

$P_{i}=\left[n\left(Y_{i}-\bar{M}\right)^{2}+\sum_{j=1}^{n}\left(Y_{i j}-\bar{Y}_{i .}-M_{j}+\bar{M}\right)^{2}\right] / 2 n$

em que:

$\mathrm{Y}_{\mathrm{i} .}=\sum_{\mathrm{j}=1}^{\mathrm{n}} \mathrm{Y}_{\mathrm{ij}} / \mathrm{n}$ : média do genótipo “i”;

$\overline{\mathrm{M}}=\sum_{\mathrm{j}=1}^{\mathrm{n}} \mathrm{M}_{\mathrm{j}} / \mathrm{n}:$ média dos genótipos com resposta

Considerando que $\mathrm{M}_{\mathrm{j}}$ representa uma cultivar hipotética, então o primeiro termo da equação representa a soma de Quadrados para o Efeito Genético, e o segundo termo é a Soma de Quadrados para a Interação Genótipos x Ambientes.

A outra metodologia utilizada foi o método proposto por Annicchiarico (1992) obtida pela expressão:

$\left.\mathrm{I}_{\mathrm{i}}=\overline{\mathrm{Y}}_{\mathrm{i}}-\mathrm{Z}_{(1-\alpha)}\right)_{\mathrm{i}}$, em que:

$\mathrm{I}_{\mathrm{i}}$ : índice de confiança (\%);

$\overline{\mathrm{Y}}_{\mathrm{i}}$ : média geral da cultivar "i" em porcentagem da média ambiental;

$\mathrm{S}_{\mathrm{i}}$ : desvio-padrão dos valores percentuais da cultivar i;

Z: percentil (1- $\alpha)$ da função de distribuição normal acumulada;

$\alpha$ : nível de significância pré-fixado; no caso $\alpha$ foi considerado como 0,25 .

\section{RESULTADOS E DISCUSSÃO}

Observou-se que a produtividade média dos experimentos nos biênios flutuou pouco, com $4.437 \mathrm{~kg} / \mathrm{ha}$ no biênio 1993/94, 4.270 kg/ha no biênio 1993/95, e $4.208 \mathrm{~kg} / \mathrm{ha}$ no biênio 1994/95. O coeficiente de variação situou-se entre 12,16\% (1994/95) e 13,68\% (1993/95), o que indica boa precisão experimental na avaliação das cultivares. Os quadrados médios de todas as fontes de variações foram significativos pelo teste $\mathrm{F}(\mathrm{P} \leq 0,01)$, à exceção do efeito de locais e da interação C x L, em todos os biênios e no triênio.

Constatou-se que no triênio a variância da interação cultivares $x$ anos dentro de locais foi $480 \%$ maior que a obtida na interação cultivares $\mathrm{x}$ locais (Tabela 2). Isso evidencia que a interação das cultivares versus anos foi mais expressiva que entre locais, o que indica a necessidade de que as avaliações de cultivares na safrinha sejam realizadas num maior número de anos. Outra alternativa importante seria que as avaliações fossem conduzidas em um maior número de épocas, dentro da safrinha, em locais mais representativos, como sugerido por Paterniani (1986) e Arias (1996). Desse modo, poderse-iam atenuar os efeitos da interação por ano, sem onerar os custos de condução dos experimentos.

O simples fato de detectar a presença da interação não contribui muito para o trabalho do melhorista. É necessário procurar meios de atenuar o efeito dessa interação. Uma das alternativas é por meio da identificação de cultivares mais adaptadas e estáveis. O conceito de adaptação adotado nesse trabalho foi o de Mariotti et al. (1976) e Ceccarelli (1996), isto é, a

TABELA 2. Resumo da análise de variância conjunta de anos e locais no que tange à produção de grãos (kg/ha), dos experimentos de avaliação de cultivares de milho-safrinha conduzidos no triênio 1993/94/95.

\begin{tabular}{lrcr}
\hline Fonte de variação & GL & QM & $\hat{\sigma}^{2}$ \\
\hline Locais (L) & 4 & 88708969,61 & \\
Ano / L & 10 & $33925149,37 * *$ & \\
R /L x A & 30 & $946283,13 * *$ & \\
Cultivar & 13 & $6620963,37 * *$ & \\
C x L & 52 & 931239,31 & 347,76 \\
C x A / L & 130 & $928109,44 * *$ & 202097,92 \\
Erro & 390 & 321815,69 & \\
\hline Média & 4449 & & \\
CV (\%) & 12,75 & & \\
\hline
\end{tabular}

** Significativo pelo teste $\mathrm{F}$ a $1 \%$ de probabilidade. 
cultivar mais adaptada será a que apresentar maior produtividade de grãos. Nesse contexto, os híbridos Zeneca 8452 (1993/94), C 805 (1993/95 e 1994/95), C 805, Agromen 2012, Agromen 2007 e C 701 (1993/94/95) foram os mais adaptados (Tabelas 3, 4, 5 e 6).

Para avaliar a estabilidade, foram consideradas as metodologias de Lin \& Binns (1988) e Annicchiarico (1992). Na primeira metodologia, é obtida a estimativa de $\mathrm{P}_{\mathrm{i}}$, que é o desvio da cultivar i em relação à que apresenta o desempenho máximo em cada local. No biênio 1993/94, o híbrido simples, Zeneca 8.452, foi o que apresentou menor estimativa de $\mathrm{P}_{\mathrm{i}}$, e a menor contribuição para a interação. Desse modo, essa cul- tivar, na maioria dos ambientes, esteve sempre nas primeiras colocações. Já os híbridos duplos AG 303, G 600, BR 201 e C 125 apresentaram as maiores estimativas de $\mathrm{P}_{\mathrm{i}}$, associados a uma maior contribuição para a interação (Tabela 3).

Pela mesma metodologia, o material que apresentou melhor desempenho no biênio 1993/95 foi o híbrido duplo Agromen 2012, e entre os de pior desempenho encontram-se os híbridos duplos C 125 e BR 201. Novamente, entre materiais que mais contribuíram para a interação encontram-se os híbridos duplo BR 201 e C 125 e o híbrido AG 519 (Tabela 4).

O destaque positivo no biênio 1994/95 foi o híbrido triplo C 805, com menor estimativa de $\mathrm{P}_{\mathrm{i}}$ e peque-

TABELA 3. Estimativas dos parâmetros de estabilidade segundo os métodos propostos por Lin \& Binns (1988) e Annicchiarico (1992) ( $\left.I_{i} \%\right)$ para produção de grãos (kg/ha), dos experimentos de avaliação de cultivares de milho-safrinha conduzidos no biênio 1993/94. Número de ambientes $=12$.

\begin{tabular}{|c|c|c|c|c|c|c|c|c|}
\hline \multirow[t]{2}{*}{ Cultivares } & \multirow{2}{*}{$\begin{array}{l}\text { Tipo de } \\
\text { cultivar }\end{array}$} & \multirow[t]{2}{*}{ Média } & \multirow{2}{*}{$\begin{array}{l}\mathrm{P}^{1} \\
(\%)\end{array}$} & \multirow[t]{2}{*}{$\mathrm{P}_{\mathrm{i}}$} & \multicolumn{2}{|c|}{ Desvio } & \multirow{2}{*}{$\begin{array}{c}\text { Contribuição } \\
\text { para interação }(\%)\end{array}$} & \multirow{2}{*}{$\begin{array}{l}\mathrm{I}_{\mathrm{i}} \\
(\%)\end{array}$} \\
\hline & & & & & Genético & Interação & & \\
\hline AG 510 & HTM $^{2}$ & 4268 & 8 & 1387,69 & 1068,84 & 318,85 & 4,99 & 89 \\
\hline AG 405 & $\mathrm{HD}^{3}$ & 4410 & 20 & 1133,43 & 871,20 & 262,23 & 4,10 & 87 \\
\hline AG 122 & $\mathrm{HD}$ & 4381 & 20 & 1241,18 & 910,58 & 330,61 & 5,17 & 90 \\
\hline AG 519 & HD & 4492 & 20 & 1136,02 & 766,53 & 369,49 & 5,78 & 92 \\
\hline AG 303 & HD & 3745 & 0 & 2452,68 & 1971,77 & 408,91 & 7,52 & 75 \\
\hline G 600 & HD & 3794 & 0 & 2195,64 & 1875,66 & 319,98 & 5,00 & 78 \\
\hline G 85 & $\mathrm{HT}^{4}$ & 4647 & 32 & 866,54 & 586,44 & 280,10 & 4,38 & 97 \\
\hline G 740 & HD & 3997 & 0 & 1808,91 & 1502,51 & 306,40 & 4,79 & 83 \\
\hline C 125 & HD & 4016 & 0 & 1943,02 & 1468,90 & 474,12 & 7,41 & 83 \\
\hline C 425 & HD & 4330 & 12 & 1239,14 & 980,70 & 258,44 & 4,04 & 91 \\
\hline C 135 & HD & 4230 & 8 & 1327,72 & 1125,00 & 202,72 & 3,17 & 88 \\
\hline XB 8028 & HD & 4563 & 24 & 907,39 & 681,24 & 226,15 & 3,54 & 95 \\
\hline Zeneca 8447 & HD & 4712 & 52 & 640,83 & 518,16 & 122,66 & 1,92 & 100 \\
\hline Agromen 2007 & HD & 4807 & 60 & 465,03 & 426,35 & 38,69 & 0,60 & 101 \\
\hline Agromen 2012 & HD & 4977 & 76 & 393,62 & 284,01 & 109,61 & 1,71 & 105 \\
\hline Agromen 2003 & HD & 4519 & 24 & 1009,02 & 733,97 & 275,05 & 4,30 & 93 \\
\hline Braskalb XL 380 & HT & 4159 & 8 & 1572,62 & 1234,91 & 337,82 & 5,28 & 84 \\
\hline C 606 & HD & 4361 & 16 & 1115,07 & 937,42 & 177,64 & 2,78 & 92 \\
\hline C 805 & HT & 4963 & 76 & 363,36 & 294,27 & 69,09 & 1,08 & 106 \\
\hline C 901 & $\mathrm{HS}^{5}$ & 4381 & 20 & 1151,04 & 910,46 & 240,58 & 3,76 & 89 \\
\hline C 701 & HD & 4952 & 76 & 414,23 & 302,77 & 111,46 & 1,74 & 102 \\
\hline DINA 70 & HD & 4436 & 20 & 1048,59 & 837,33 & 211,26 & 3,30 & 91 \\
\hline DINA 766 & HS & 4345 & 16 & 1265,81 & 959,57 & 306,23 & 4,79 & 88 \\
\hline BR 201 & HD & 4045 & 0 & 1947,46 & 1421,02 & 526,44 & 8,23 & 80 \\
\hline Zeneca 8452 & HS & 5390 & 96 & 96,18 & 58,06 & 38,12 & 0,60 & 113 \\
\hline \multicolumn{2}{|l|}{ Média } & \multicolumn{5}{|l|}{4437} & & \\
\hline $\begin{array}{ll}1 & \text { Porcentagem de cu } \\
2 & \text { HTM: híbrido tripl } \\
3 & \text { HD: híbrido duplo. } \\
& \\
& \text { HT: híbrido triplo. } \\
5 & \text { HS: híbrido simple }\end{array}$ & $\begin{array}{l}\text { res que for: } \\
\text { dificado. }\end{array}$ & superadas & atistic & nte pela cult & pelo teste de & ncan a $5 \%$ de & robabilidade. & \\
\hline
\end{tabular}


na contribuição para a interação. Os híbridos com maiores estimativas de $\mathrm{P}_{\mathrm{i}}$, e com grande contribuição para a interação, e, portanto, mais instáveis, foram o híbrido duplo Agromen 2003 e o híbrido triplo Braskalb XL 380 (Tabela 5). Os resultados do triênio 1993/94/95 encontram-se na Tabela 6. A cultivar que se destacou com melhor desempenho foi Agromen 2012, e as de pior, a C 125, a Braskalb XL 380 e a BR 201.

A segunda metodologia utilizada foi a Annicchiarico (1992), que considera que toda atividade agrícola envolve algum risco. Desse modo, a escolha de uma dada cultivar, para ser recomendada aos agricultores, será feita a partir da estimativa do índice de confiança $\left(\mathrm{I}_{\mathrm{i}}\right)$, que considera o risco de uma dada cultivar. As estimativas dos valores obtidos no biênio 1993/94 são apresentadas na Tabela 3. Constatou-se que também por essa metodologia o híbrido Zeneca 8452 foi o destaque, pois com $75 \%$ de probabilidade, na pior das hipóteses, a sua produtividade será $13 \%$ acima da média do ambiente, sendo, portanto, o de maior confiabilidade. Já o con- trário ocorreu com o AG 303, cuja produtividade média esperada pode chegar a ser $25 \%$ abaixo da média do ambiente.

Coerentemente com a estimativa de Lin \& Binns (1988), no biênio 1993/95 os materiais que apresentaram maiores índices de confiança foram os híbridos Agromen 2012 e C 805, cujo desempenho, na pior das hipóteses, será $7 \%$ acima da média do ambiente. Os materiais Braskalb XL 380 e C 125 apresentaram índice de confiança inferior a 1 (Tabela 4).

Pelos resultados obtidos no biênio 1994/95, o híbrido C 805 mostrou ser o de menor risco. Com $75 \%$ de probabilidade, sua produtividade deve ficar, na pior das hipóteses, $6 \%$ acima da média do ambiente. As menores estimativas do índice de confiança foram dos híbridos Agromen 2003 e Planagri 401, cujo desempenho esperado pode ser até $20 \%$ abaixo da média do ambiente (Tabela 5). Na análise dos dados do triênio 1993/94/95, os híbridos Agromen 2012 e C 805 foram os que apresentaram maiores estimativas do índice de confiança, e C 125 e BR 201 foram os de maiores riscos para os agricultores (Tabela 6).

TABELA 4. Estimativas dos parâmetros de estabilidade segundo os métodos proposto por Lin \& Binns (1988) e Annicchiarico (1992) $\left(I_{i} \%\right)$ quanto à produção de grãos (kg/ha), dos experimentos de avaliação de cultivares de milho-safrinha conduzidos no biênio 1993/95. Número de ambientes $=12$.

\begin{tabular}{|c|c|c|c|c|c|c|c|c|}
\hline \multirow[t]{2}{*}{ Cultivares } & \multirow{2}{*}{$\begin{array}{l}\text { Tipo de } \\
\text { cultivar }\end{array}$} & \multirow[t]{2}{*}{ Média } & \multirow{2}{*}{$\begin{array}{l}\mathrm{P}^{1} \\
(\%)\end{array}$} & \multirow[t]{2}{*}{$P_{i}$} & \multicolumn{2}{|c|}{ Desvio } & \multirow{2}{*}{$\begin{array}{c}\text { Contribuição } \\
\text { para interação }(\%)\end{array}$} & \multirow{2}{*}{$\begin{array}{l}\mathrm{I}_{\mathrm{i}} \\
(\%)\end{array}$} \\
\hline & & & & & Genético & Interação & & \\
\hline AG 122 & $\mathrm{HD}^{2}$ & 4097 & 20 & 850,36 & 637,41 & 212,95 & 8,40 & 87 \\
\hline AG 519 & $\mathrm{HD}$ & 4238 & 47 & 864,14 & 488,15 & 375,99 & 14,83 & 90 \\
\hline G 85 & $\mathrm{HT}^{3}$ & 4405 & 53 & 564,52 & 337,09 & 227,43 & 8,97 & 94 \\
\hline C 125 & HD & 3712 & 0 & 1462,98 & 1146,86 & 316,13 & 12,47 & 75 \\
\hline XB 8030 & HD & 4403 & 53 & 466,66 & 338,66 & 128,00 & 5,05 & 96 \\
\hline Agromen 2007 & HD & 4662 & 73 & 228,22 & 159,47 & 68,75 & 2,71 & 102 \\
\hline Agromen 2012 & HD & 4784 & 93 & 140,33 & 97,79 & 42,54 & 1,68 & 107 \\
\hline Agromen 2003 & HD & 4389 & 53 & 465,53 & 350,21 & 115,32 & 4,55 & 93 \\
\hline AL 25 & $\mathrm{~V}^{4}$ & 4022 & 20 & 877,13 & 725,21 & 151,92 & 5,99 & 91 \\
\hline Braskalb XL 380 & HT & 3847 & 7 & 1107,89 & 951,51 & 156,38 & 6,17 & 75 \\
\hline C 606 & HD & 4143 & 27 & 726,91 & 586,81 & 140,11 & 5,53 & 91 \\
\hline C 805 & HT & 4795 & 93 & 211,57 & 92,84 & 118,72 & 4,68 & 107 \\
\hline C 901 & $\mathrm{HS}^{5}$ & 4079 & 20 & 706,84 & 658,76 & 48,08 & 1,90 & 82 \\
\hline C 701 & HD & 4580 & 73 & 284,37 & 208,66 & 75,72 & 2,99 & 100 \\
\hline BR 201 & HD & 3886 & 7 & 1255,39 & 898,69 & 356,70 & 14,07 & 82 \\
\hline Média & & 4270 & & & & & & \\
\hline \multicolumn{9}{|c|}{$\begin{array}{l}{ }^{1} \text { Porcentagem de cultiv } \\
2 \\
\text { HD: híbrido duplo. } \\
{ }^{3} \text { HT: híbrido triplo. } \\
{ }^{4} \text { V: variedade. } \\
{ }^{5} \text { HS: híbrido simples. }\end{array}$} \\
\hline
\end{tabular}


TABELA 5. Estimativas dos parâmetros de estabilidade segundo os métodos proposto por Lin \& Binns (1988) e Annicchiarico (1992) ( $\left.I_{i} \%\right)$ quanto à produção de grãos $(\mathrm{kg} / \mathrm{ha})$, dos experimentos de avaliação de cultivares de milho-safrinha conduzidos no biênio 1994/95. Número de ambientes $=12$.

\begin{tabular}{|c|c|c|c|c|c|c|c|c|}
\hline \multirow[t]{2}{*}{ Cultivares } & \multirow{2}{*}{$\begin{array}{l}\text { Tipo de } \\
\text { cultivar }\end{array}$} & \multirow[t]{2}{*}{ Média } & \multirow{2}{*}{$\begin{array}{l}\mathrm{P}^{1} \\
(\%)\end{array}$} & \multirow[t]{2}{*}{$\mathrm{P}_{\mathrm{i}}$} & \multicolumn{2}{|c|}{ Desvio } & \multirow{2}{*}{$\begin{array}{c}\text { Contribuição } \\
\text { para interação (\%) }\end{array}$} & \multirow{2}{*}{$\begin{array}{l}\mathrm{I}_{\mathrm{i}} \\
(\%)\end{array}$} \\
\hline & & & & & Genético & Interação & & \\
\hline C 701 & $\mathrm{HD}^{2}$ & 4331 & 19 & 593,12 & 457,53 & 135,59 & 2,62 & 90 \\
\hline C 901 & $\mathrm{HS}^{3}$ & 4017 & 0 & 977,61 & 806,34 & 171,27 & 3,31 & 89 \\
\hline C 805 & $\mathrm{HT}^{4}$ & 4750 & 96 & 242,45 & 144,32 & 98,13 & 1,90 & 106 \\
\hline C 606 & $\mathrm{HD}$ & 4065 & 0 & 934,98 & 747,46 & 187,52 & 3,63 & 91 \\
\hline CO 822992 & -- & 4260 & 12 & 835,28 & 527,96 & 307,32 & 5,94 & 90 \\
\hline Agromen 2012 & HD & 4345 & 19 & 649,56 & 444,07 & 205,49 & 3,97 & 94 \\
\hline Agromen 2007 & HD & 4330 & 19 & 565,11 & 457,69 & 107,42 & 2,08 & 98 \\
\hline Agromen 2003 & HD & 3820 & 0 & 1401,32 & 1076,53 & 324,79 & 6,28 & 77 \\
\hline Planagri 401 & HD & 4387 & 31 & 512,54 & 405,38 & 107,17 & 2,07 & 78 \\
\hline Planagri 402 & -- & 4249 & 12 & 820,32 & 538,89 & 281,43 & 5,44 & 90 \\
\hline AG 122 & HD & 4411 & 31 & 525,94 & 384,27 & 141,67 & 2,74 & 98 \\
\hline BR 205 & HD & 3857 & 0 & 962,83 & 884,23 & 78,61 & 1,52 & 83 \\
\hline C 125 & HD & 4174 & 8 & 910,39 & 619,48 & 290,91 & 5,63 & 86 \\
\hline C 435 & HD & 4056 & 0 & 902,26 & 758,30 & 143,97 & 2,78 & 91 \\
\hline G 85 & HT & 4304 & 15 & 619,07 & 483,72 & 135,35 & 2,62 & 96 \\
\hline G 550 & HT & 4293 & 15 & 667,00 & 494,18 & 172,82 & 3,34 & 92 \\
\hline Braskalb xl 380 & HT & 4140 & 8 & 1142,12 & 658,00 & 484,12 & 9,36 & 80 \\
\hline Braskalb XL 660 & HD & 4252 & 12 & 693,35 & 535,61 & 157,73 & 3,05 & 92 \\
\hline Braskalb XL 655 & HD & 4248 & 12 & 894,23 & 539,59 & 354,64 & 6,86 & 88 \\
\hline Planagri 411 & HD & 4124 & 4 & 902,98 & 676,38 & 226,59 & 4,38 & 90 \\
\hline AL 25 & $\mathrm{~V}^{5}$ & 3934 & 0 & 1046,55 & 915,19 & 131,36 & 2,54 & 87 \\
\hline AG 1043 & HD & 4286 & 15 & 568,71 & 501,67 & 67,05 & 1,30 & 97 \\
\hline AG 951 & HT & 3981 & 0 & 1195,60 & 852,49 & 343,11 & 6,64 & 81 \\
\hline AG 519 & HD & 4394 & 31 & 504,25 & 399,32 & 104,92 & 2,03 & 96 \\
\hline BR 201 & HD & 4226 & 12 & 852,14 & 562,95 & 289,19 & 5,59 & 91 \\
\hline BR 206 & HD & 4067 & 0 & 867,44 & 744,91 & 122,53 & 2,37 & 88 \\
\hline Média & & 4208 & & & & & & \\
\hline \multicolumn{9}{|c|}{$\begin{array}{l}1 \text { Porcentagem de cultiva } \\
2 \text { HD: híbrido duplo. } \\
3 \text { HS: híbrido simples. } \\
4 \text { HT: híbrido triplo. } \\
5 \text { V: variedade. }\end{array}$} \\
\hline \multicolumn{9}{|c|}{$\begin{array}{l}\text { TABELA 6. Estimativas dos parâmetros de estabilidade segundo os métodos proposto por Lin \& Binns (1988) } \\
\text { e Annicchiarico }(1992)\left(I_{i} \%\right) \text { quanto à produção de grãos (kg/ha), dos experimentos de avaliação de } \\
\text { cultivares de milho-safrinha conduzidos no triênio1993/94/95. Número de ambientes }=15 \text {. }\end{array}$} \\
\hline \multirow[t]{2}{*}{ Cultivares } & \multirow{2}{*}{$\begin{array}{l}\text { Tipo de } \\
\text { cultivar }\end{array}$} & \multirow[t]{2}{*}{ Média } & \multirow{2}{*}{$\begin{array}{l}\mathrm{P}^{1} \\
(\%)\end{array}$} & \multirow[t]{2}{*}{$\mathrm{P}_{\mathrm{i}}$} & \multicolumn{2}{|c|}{ Desvio } & & $\mathrm{I}_{\mathrm{i}}$ \\
\hline & & & & & Genético & Interação & para interação (\%) & $(\%)$ \\
\hline AG 122 & $\mathrm{HD}^{2}$ & 4377 & 21 & 791,05 & 580,04 & 211,01 & 8,89 & 89 \\
\hline AG 519 & HD & 4535 & 29 & 715,03 & 422,16 & 292,87 & 12,34 & 93 \\
\hline G 85 & $\mathrm{HT}^{3}$ & 4730 & 57 & 465,23 & 262,14 & 203,09 & 8,55 & 98 \\
\hline C 125 & HD & 3898 & 0 & 1488,51 & 1211,50 & 277,01 & 11,67 & 78 \\
\hline Agromen 2007 & HD & 4802 & 64 & 284,93 & 212,42 & 72,51 & 3,05 & 102 \\
\hline Agromen 2012 & HD & 4984 & 64 & 202,93 & 110,36 & 92,57 & 3,90 & 106 \\
\hline Agromen 2003 & HD & 4469 & 29 & 662,83 & 485,31 & 177,53 & 7,48 & 91 \\
\hline AL 25 & $\mathrm{~V}^{4}$ & 4131 & 0 & 1005,28 & 875,69 & 129,58 & 5,46 & 88 \\
\hline Braskalb XL 380 & HT & 3919 & 0 & 1383,55 & 1178,93 & 204,62 & 8,62 & 76 \\
\hline С 606 & HD & 4278 & 21 & 843,63 & 691,33 & 152,30 & 6,42 & 90 \\
\hline C 805 & HT & 4983 & 64 & 226,61 & 111,14 & 115,47 & 4,86 & 106 \\
\hline
\end{tabular}


TABELA 6. Continuação.

\begin{tabular}{|c|c|c|c|c|c|c|c|c|}
\hline \multirow[t]{2}{*}{ Cultivares } & \multirow{2}{*}{$\begin{array}{l}\text { Tipo de } \\
\text { cultivar }\end{array}$} & \multirow[t]{2}{*}{ Média } & \multirow{2}{*}{$\begin{array}{l}\mathrm{P}^{1} \\
(\%)\end{array}$} & \multirow[t]{2}{*}{$P_{i}$} & \multicolumn{2}{|c|}{ Desvio } & \multirow{2}{*}{$\begin{array}{c}\text { Contribuição } \\
\text { para interação }(\%)\end{array}$} & \multirow{2}{*}{$\begin{array}{c}\mathrm{I}_{\mathrm{i}} \\
(\%)\end{array}$} \\
\hline & & & & & Genético & Interação & & \\
\hline C 901 & $\mathrm{HS}^{5}$ & 4345 & 21 & 703,84 & 615,61 & 88,24 & 3,72 & 90 \\
\hline C 701 & HD & 4964 & 64 & 253,63 & 174,40 & 79,23 & 3,34 & 102 \\
\hline BR 201 & HD & 3974 & 0 & 1373,58 & 1095,59 & 277,99 & 11,71 & 80 \\
\hline Média & & 4449 & & & & & & \\
\hline
\end{tabular}

${ }^{1}$ Porcentagem de cultivares que foram superadas estatisticamente pela cultivar i, pelo teste de Duncan a 5\% de probabilidade.

HD: híbrido duplo.

HT: híbrido triplo.

V: variedade

HS: híbrido simples.

Finalmente, é oportuno salientar que, como se constatou, as duas metodologias utilizadas na avaliação da adaptabilidade e estabilidade forneceram praticamente os mesmos resultados. Ambas são de fácil aplicação e interpretação.

\section{CONCLUSÕES}

1. A interação das cultivares com anos dentro de locais foi mais expressiva do que entre locais, o que indica a necessidade de que as avaliações de cultivares na safrinha sejam realizadas num maior número de anos.

2. Os híbridos Zeneca 8452, C 805 e Agromen 2012 são mais estáveis, com menor risco para os agricultores em safrinha. Os de maiores risco são C 125 , AG 303, BR 201 e Planagri 411.

\section{AGRADECIMENTOS}

A Embrapa-Centro Nacional de Pesquisa de Milho e Sorgo (CNPMS), por ter cedido os dados para que este trabalho pudesse ser realizado.

\section{REFERÊNCIAS}

ANNICCHIARICO, P. Cultivar adaptation and recommendation from alfalfa trials in Northern Italy. Journal of Genetics \& Breeding, v.46, n.1, p.269278, Mar. 1992.

ARIAS, E.R.A. Adaptabilidade e estabilidade das cultivares de milho avaliadas no Estado de Mato Grosso do Sul e avanço genético obtido no período de 1986/87 a 1993/94. Lavras: UFLA, 1996. 118p. Tese de Doutorado.
CECCARELLI, S. Adaptation to low/high input cultivation. Euphytica, Wageningen, v.92, n.2, p.203214, Dec. 1996.

CRUZ, C.D.; REGAZZI, A.J. Modelos biométricos aplicados ao melhoramento genético. Viçosa, MG: UFV, 1994. 390p.

CRUZ, C.D.; TORRES, A. de A.R.; VENCOVSKY, R. An alternative approach to the stability analysis proposed by Silva and Barreto. Revista Brasileira de Genética, Ribeirão Preto, v.12, n.2, p.567-580, 1989.

EBERHART, S.A.; RUSSEL, W.A. Stability parameters for comparing varieties. Crop Science, Madison, v.6, n.1, p.36-40, 1966.

ENCICLOPÉDIA dos Municípios Brasileiros. Rio de Janeiro: IBGE, 1958. v.12, p.267-271, v.13, p.201206, v.15, p.119-122, 269-270, 346-347, v. 16, p.244245, v.17, p.90-93.

LIN, C.S.; BINNS, M.R. A superiority measure of cultivar performance for cultivar x location data. Canadian Journal of Plant Science, Ottawa, v.68, n.1, p.193-198, Jan. 1988.

MARIOTTI, J.A.; OYARZABAL, E.S.; OSA, J.M.; BULACIO, A.N.R.; ALMADA, G.H. Análisis de estabilidad y adaptabilidad de genótipos de caña de azucar. I. Interacciones dentro de una localidad experimental. Revista Agronómica del NoroesteArgentino, Tucuman, v.13, n.1/4, p.105-127, Jan. 1976.

PATERNIANI, E. Interação genótipo $\mathrm{x}$ ambiente em climas tropicais e sub-tropicais. In: CONGRESSO NACIONAL DE MILHO E SORGO, 16., 1986, Belo Horizonte. Anais... Sete Lagoas: EmbrapaCNPMS, 1986. p.378-382. 